\title{
Findings of Somatosensory Evoked Potential to Stimulation of the Sciatic Nerve in Two Different Rat Strains
}

\author{
Atilla OǦUZHANOǦLU and Tülay KURT \\ Department of Neurology, Faculty of Medicine, Pamukkale University, Denizli, 20100 Turkey
}

\begin{abstract}
No comparative study about somatosensory evoked potentials (SEP) on different rat strains has been done yet. It is evident that comparative SEP studies are important since different rat strains have different physiological properties. We aimed to compare early latency SEP values from stimulation of sciatic nerve in Wistar $(W r)$ and Sprague-Dawley (SDr) rats which are frequently used rat strains in experimental studies. In Wr group, the mean of first far field potential (Ff1) latency was shorter and the mean Ff1 amplitude was lower than that of Sprague-Dawley rat group. Mean cortical potential latency in Wr group was longer than that of SDr group while amplitude was not different. Central conduction time (CCT) in Wistar rat group was found to be longer than that of SDr group. Shorter Ff1 latency in Wr group implies that afferent volley reaches cervical posterior fasciculus from sciatic nerve earlier than SDr group while longer CP latency implies that afferent volley reaches cortex later than SDr group. Similarity between the latencies of lumbar potentials implies that peripheral conduction velocity has no effect on the difference of Ff1 latencies.
\end{abstract}

Key words: central conduction time, somatosensory evoked potentials, Sprague-Dawley rat, Wistar rat

\section{Introduction}

There are many studies concerning somatosensory evoked potential (SEP), however no comparative study on the SEP of different rat strains exists and it is evident that comparative SEP studies are important since different rat strains have different physiological properties.

Therefore we aimed to compare early latency SEP values after stimulation of the sciatic nerve in Wistar(Wr) and Sprague-Dawley (SDr) rats which are frequently used rat strains in experimental studies.

\section{Materials and Methods}

The female rats investigated were 10-12 weeks old and a total of $12 \mathrm{Wr}$ and $12 \mathrm{SDr}$ rats were included in the study. Both groups were kept under the same conditions, i.e. temperature was between $22-25^{\circ} \mathrm{C}$, relative humidity was about $50 \%$, and the light-dark cycle was 12:12 hr. During and after the study none of the animals were killed or disabled. Recordings were done under general anesthesia which was achieved by injecting intraperitoneally a mixture of $12 \mathrm{mg} / \mathrm{kg}$ xylazine and $75 \mathrm{mg} / \mathrm{kg}$ ketamine. Two-channel recording was 
Table 1. Mean distance from sciatic notch to active (Cz) electrode and mean weight in two groups

\begin{tabular}{lccl}
\hline Strain (n) & Dist (Mean-S.D.) (cm) & \multicolumn{2}{c}{ Weight (Mean-S.D.) (gr) } \\
\hline Wr (12) & $13.083(1.062)$ & 195 & $(17.581)$ \\
SDr (12) & $12.875(0.98)$ & $187.08(10.54)$
\end{tabular}

Dist: Distance, Wr: Wistar rats, SDr: Sprague-Dawley rats, S.D.: Standard deviation.

also performed on 4 new animals for each group to display the temporal relationship between lumbar and scalp potentials. Thr mean distance from the stimulation point (where the sciatic nerve emerges from the pelvis-sciatic notch) to the active electrode $(\mathrm{Cz})$ and the mean weight of rats are shown in Table 1. There were no differences between the two strains for those parameters.

For scalp SEP recordings, the $\mathrm{C}_{\mathrm{z}}$ electrode (placed about $5 \mathrm{~mm}$ behind the bregma on the midline) was referenced to the $F_{z}$ electrode (placed about $5 \mathrm{~mm}$ in front of the bregma on the midline) both of which were monopolar needle electrodes. For lumbar potential (LP) recordings, a $\mathrm{L}_{1}$ (placed on the first lumbar vertebra) monopolar disc electrode was referenced to $\mathrm{T}_{11}$ (placed on the eleventh thoracal vertebra). For ground, a needle monopolar electrode was used and inserted into the tail. A coaxial needle was used for stimulation and the left sciatic nerve was stimulated deeply at the sciatic notch where it emerges from the pelvis. Stimulus intensity was kept at a level which resulted in slight twitching of hind limb fingers.

SEPs were recorded using a Medelec (Premiere Plus) electromyography device. The frequency bandwidth of the amplifier was $0.1 \mathrm{~Hz}-10 \mathrm{kHz}$. Sensitivity level was $100 \mu \mathrm{V} /$ division for scalp recordings and $1 \mathrm{mV} / \mathrm{div}$. for lumbar recordings. Impedance was maintained below $2 \mathrm{k} \Omega$. Stimulus frequency was five times a second with a duration of $500 \mu \mathrm{s}$, and its intensity was about $1.9 \mathrm{~mA}$. Sweep time was $30 \mathrm{~ms}$. Five hundred and twelve responses were averaged and this trial was repeated to ensure the replicability of findings. Means of the values attained on two traces were used to measure latency and amplitude. Laboratory temperature was between $32-33^{\circ} \mathrm{C}$ during the experiments.

Data obtained were evaluated on Macintosh PPC computer using the Statview 512 program and a t-test for unpaired groups.

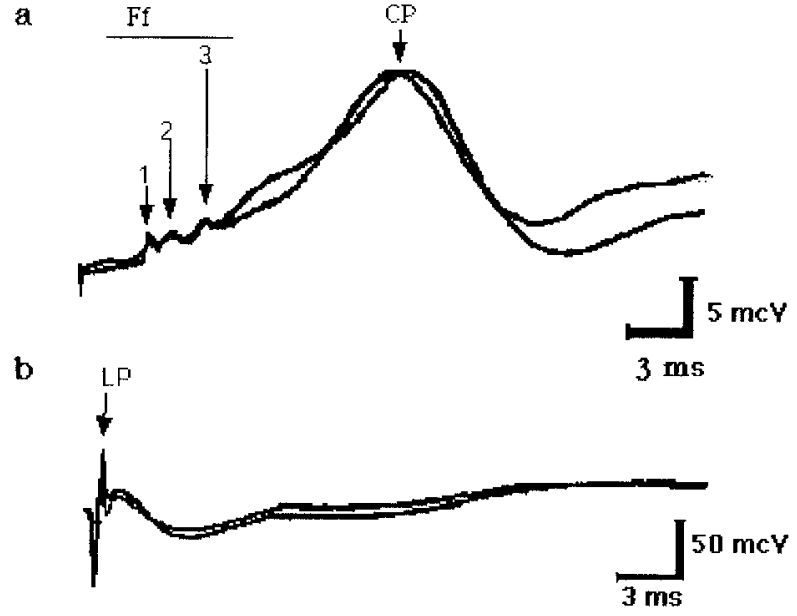

Fig. 1. Cortical potentials (a) and simultaneously recorded lumbar potential (b) with $30 \mathrm{~ms}$ sweep time. Two traces of averaged 512 responses. Ff: Far field potentials, CP: Somatosensory cortex potential, LP: Lumbar potential.

\section{Results}

Absolute latencies of the first negative deflection of far-field potentials (Ff1) and cortical potential (CP), as well as their peak to peak amplitudes, were measured on the recorded traces (Fig. 1a). Table 2 shows the mean and standard deviations of these values. In $\mathrm{Wr}$ group, the mean $\mathrm{Ff}_{1}$ latency was shorter $(\mathrm{t}=-3.60$, $\mathrm{p}=0.0016)$ and the mean $\mathrm{Ff}_{1}$ amplitude was lower ( $\mathrm{t}=-$ 2.809, $\mathrm{p}=0.0102$ ) than those of the SDr group. The mean CP latency in the $\mathrm{Wr}$ group was longer $(\mathrm{t}=5.101$, $\mathrm{p}=0.0001$ ) than that of the SDr while the amplitude was not different.

Central conduction time in the $\mathrm{Wr}$ group, calculated as subtraction of the aforementioned potentials (in this sample it means latency difference from fasciculus gracilis-Ff1- to somatosensory cortex-Cp), was found to be longer than that of the SDr group.

Two-channel simultaneous recordings performed on four new $\mathrm{Wr}$ and four new SDr to show the relationship between LP and scalp recorded potentials are seen in Fig. 1b. Mean latency differences between Ff1 and $\mathrm{LP}$ were $2.51 \mathrm{~ms}$ for $\mathrm{Wr}$ and $2.93 \mathrm{~ms}$ for SDr. These two means were not significantly different.

\section{Discussion}

Evoked potentials (EP) have aroused investigator's 
Table 2. Means and standard deviations of the first arrival of the far field and somatosensory cortex potentials of two rat strains

\begin{tabular}{lllllc}
\hline & \multicolumn{2}{c}{ Far field 1 (Mean-S.D.) } & \multicolumn{2}{c}{ CP (Mean-S.D.) } & CCT \\
& Lat $(\mathrm{ms})$ & Amp $(\mu \mathrm{V})$ & Lat $(\mathrm{ms})$ & Amp $(\mu \mathrm{V})$ & $(\mathrm{ms})$ \\
\hline $\mathrm{Wr}(12)$ & $4.32(0.30)$ & $1.26(1.02)$ & $19.88(1.64)$ & $6.11(1.55)$ & $15.56(1.72)$ \\
SDr $(12)$ & $4.69(0.20)^{* *}$ & $2.29(0.75)^{*}$ & $17.39(0.90)^{* *}$ & $6.60(2.98)$ & $12.69(0.90)^{* *}$ \\
\hline
\end{tabular}

Wr: Wistar rats, SDr: Sprague-Dawley rats, Lat: Latency, Amp: Amplitude, Far field1: The first arrival of the far field potentials, CP: Cortical potential, CCT: Central conduction time, S.D.: Standard deviation, ms: millisecond, $\mu \mathrm{V}$ : micro volt. Significantly different from Wr. ${ }^{*}: \mathrm{p}<0.05$, **: $\mathrm{p}<0.01$

interest for more than a century as reported by Aminoff and Eisen [3]. There are many studies about EP in humans as well as animals, but there is no study to compare SEP findings on different laboratory rat strains. SEPs and their neural generators are described in many reports $[3,4-6]$.

Scalp recordings show not only cortex-generated potentials, but also far field (Ff) potentials which are generated by structures far from the active electrode and reach the recording site by volume conduction. Generators of far field potentials were shown by Wiederholt \& Iragui-Madoz [12], and also SEPs in four species were shown by Allison and Hume [2] to be remarkably similar in morphology and surface topography.

The first of the far field potentials $\left(\mathrm{Ff}_{1}\right)$ originates in the cervical posterior column (fasciculus gracilis) in rats, as Wiederholt and Iragui-Madoz [12] observed, and the existence of a similar potential was also revealed in humans $[1,2,7]$. It was observed that Ff1 was still recorded even following transection at the midbrain $[10,12]$ or the medullospinal junction [12]. A high amplitude negative potential on bipolar recordings (Fig. 1, CP) is assumed to originate in the somatosensory cortex.

Measurement of interpeak latencies obtained on scalprecorded traces present more reliable data than that of absolute latencies. Central conduction time (CCT) [3, $5,11]$ is a kind of interpeak latency measurement and gives information about intracranial lesions.

In the present study, it was found that $\mathrm{Ff}_{1}$ latency in the Wr group was shorter and its amplitude was lower than that of the SDr group. In addition, CP latency of the $\mathrm{Wr}$ group was longer than that of the SDr group. Central conduction time (interpeak latency) in the $\mathrm{Wr}$ group was found to be longer than that of the SDr group. A shorter $\mathrm{Ff}_{1}$ latency in the $\mathrm{Wr}$ group implies that the afferent volley reaches the cervical posterior fasciculus from the sciatic nerve earlier than in the SDr group, while a longer CP latency implies that the afferent volley reaches the cortex later than in the SDr group. This means that the electrical stimulus travel faster through the spinal cord in the $\mathrm{Wr}$ than in the SDr while it travels slower through cerebrum in the $\mathrm{Wr}$ than in the SDr. Similarity between the latencies of LPs implies that peripheral conduction has no effect on the difference of the $\mathrm{Ff}_{1}$ latencies. Further studies are needed to confirm these SEP differences between the two rat strains.

Anesthesia significantly influenced the cortical components of the SEP, as stated by Koyanagi and Tator [9], but Goss-Sampson and Kriss [8] reported that the combination of ketamine and xylazine, which we used, did not affect sensory or motor conduction and interpeak latencies.

In conclusion, stimulation of the sciatic nerve and bipolar scalp recordings showed latency and amplitude differences for $\mathrm{Ff}_{1}$ potential and latency differences for somatosensory cortex potential between Wistar and Sprague-Dawley rats. Further studies are needed to investigate the origin of these differences.

\section{References}

1. Abbruzzese, M., Favale, E., and Leandri, M. 1978. Spinal components of the cerebral somatosensory evoked response in normal man: The "S wave". Acta. Neurol. Scandinav. 58: $213-220$.

2. Allison, T. and Hume, A.L. 1981. A Comparative analysis of short-latency somatosensory evoked potentials in man, monkey, cat, and rat. Exp. Neuro. 72: 592-611.

3. Aminoff, M.J. and Eisen, A. 1992. Somatosensory Evoked Potentials. pp. 571-603. In: Electrodiagnosis in Clinical Neurology (Aminoff MJ ed.), Churchill Livingstone, New 
York.

4. Chiappa, K.H. 1990. Short-Latency Somatosensory Evoked Potentials: Methodology. pp. 307-435. In: Evoked Potentials in Clinical Medicine (Chiappa. K.H. ed.), Raven Press, New York.

5. Eisen, A. 1982. The Somatosensory Evoked Potential. Can. J. Neurol. Sci. 9: 65-77.

6. Emerson, R.G. 1988. Anatomic and physiologic bases of posterior tibial nerve somatosensory evoked potentials. Neurol. Clin. 6: 735-749.

7. Ertekin, C. 1976. Studies on the human evoked electrospinogram. Acta. Neurol. Scandinav. 53: 3-20.

8. Goss-Sampson, M.A. and Kriss, A. 1991. Effects of pentobarbital and ketamine-xylazine anaesthesia on somatosensory, brainstem auditory and peripheral sensorymotor responses in the rat. Lab. Anim. 25: 360-366.
9. Koyanagi, I. and Tator, C.H. 1996. The effects of cortical stimulation, anesthesia and recording site on somatosensory evoked potentials in the rat. Electroenceph. Clin. Neurophysiol. 101: 534-542.

10. Sen, C.N. and Maller, A.R. 1991. Comparison of somatosensory evoked potentials recorded from the scalp and dorsal column nuclei to upper and lower limb stimulation in the rat. Electroenceph. Clin. Neurophysiol. 80: $378-383$.

11. Shaw, N.A. 1992. The effects of low pass filtering on central somatosensory conduction time. Brain Res. Bull. 28: 803809.

12. Wiederholt, W.C. and Iragui-Madoz, V.J. 1977. Far field somatosensory evoked potentials in the rat. Electroenceph. Clin. Neurophysiol. 42: 456-465. 